\title{
A Diamond Cell for X-ray Diffraction Studies at High Pressures
}

\author{
G. J. Piermarini and C. E. Weir
}

(April 5, 1962)

\begin{abstract}
A high pressure X-ray powder camera has been constructed. The instrument has been found to be useful for routine X-ray work, using molybdenum radiation, to pressures of approximately 60 kilobars. Previously reported transitions have been observed in silver iodide, potassium iodide, bismuth, and thallium. The high pressure forms and lattice parameters were found to be: $\mathrm{AgI}$ - f.c.c. ( $\mathrm{NaCl}$ type), $a_{0}=6.067 \mathrm{~A}$; KI - s.c. (CsCl type), $a_{0}=4.093 \mathrm{~A}$; Tl-f.c.c. (NaCl type), $a_{0}=4.778 \mathrm{~A}$; Bi structure not determined. These data confirm previous reports on the high pressure forms of $\mathrm{AgI}$ and $\mathrm{KI}$. Data on $\mathrm{Tl}$ and Bi are apparently reported for the first time. The high pressure modifications were studied at the following approximate pressures which are not indicative of the point where the transition occurs: AgI - 3.3 kilobars, KI-20 kilobars, Bi -28 kilobars, and Tl-60 kilobars.

The pressure limit to which the unit can be used successfully has not been ascertained. It is believed to be much higher than the pressures reported. The present instrument is capable of producing powder diffraction patterns of materials of relatively high scattering power, giving data to $2 \theta=35^{\circ}$. High background on the X-ray powder patterns is believed to arise from scattering by the diamonds. This background may obscure weak diffraction rings. This effect may be reduced by screening, monochromatization, and other improvements in experimental technique.
\end{abstract}

\section{Introduction}

There has beeen considerable interest in the structures of high pressure polymorphs ever since Bridgman's studies showed the frequency of occurrence of transitions at elevated pressures [1]. ${ }^{1}$ High pressure forms are not usually obtained by cooling at $1 \mathrm{~atm}$, and in only a few instances appear to be obtainable in a metastable condition at $1 \mathrm{~atm}$. McFarlan $[2,3]$ used this method of determining the X-ray structure of certain of the high pressure forms of ice. In general, it appears that the structures of the high pressure polymorphs can be studied only at high pressure. The first successful attack on this problem appears to have been made by Jacobs $[4,5]$ who obtained powder patterns of high pressure phases with a camera enclosed in the pressure vessel. $\mathrm{He}$ used helium gas as the pressure-transmitting fluid and a beryllium window to permit $\mathrm{X}$-rays to enter the vessel. This method was used to a maximum pressure of 5 kilobars $\left(1\right.$ bar $=10^{6}$ dynes $\left.\mathrm{cm}^{-2}\right)$. The next marked advance appears to have been made by Lawson and his coworkers who constructed first a miniature beryllium pressure vessel [6] and later a miniature diamond pressure vessel [7]. The vessels were transparent to Mo K X-rays and the enclosed specimen could be studied. Using diamonds the maximum pressure attainable appears to have been approximately 30 kilobars. The diamond cell method was used by Jamieson [8, 9] for studies of high pressure polymorphs of geological interest. Jamieson later $[10,11]$ has reported a high pressure X-ray

${ }^{1}$ Figures in brackets indicate the literature references at the end of this paper. device using diamonds in an anvil-type squeezer. In this technique the flat specimen was loaded to a desired stress between two single crystal diamond pistons. This unit was designed to be mounted on the spindle of an X-ray diffractometer. A similar device is being studied by Adams [12]. Evdokimova and Vereshchagin [13] have used an externally supported beryllium pressure vessel to study the lattice parameters of strontium and barium to a maximum pressure of 15 kilobars.

Recently an infrared cell using diamonds in an anvil-type squeezer was developed in this laboratory [14] and used successfully for studies to pressures of 50 kilobars in a routine manner. The design of this cell has been modified to permit studies of X-ray powder diffraction patterns to pressures of at least 60 kilobars. This report describes the instrument and preliminary studies on a few materials of interest which serve to illustrate its range of application.

\section{Apparatus}

The high pressure X-ray camera is shown in cross section in figure 1 . The unit contains a hardened steel entablature $(\mathrm{E})$ and a piston $(\mathrm{H})$ actuated by hydraulic pressure generated by means of an external hand pump and introduced through a fitting (M). The hydraulic liquid is confined to the small volume between the rubber $\mathrm{O}$-rings $(\boldsymbol{J})$ and exerts an axial thrust on the shoulder of the piston $(\mathrm{H})$. The entablature (E) is supported by the thrust washer (C) and the cap (B) which screws on to the unit. Both the entablature and the piston are fitted with small brilliant-cut gem-quality diamonds (F) each weighing approximately $0.03 \mathrm{~g}$ (or 0.15 carats). The 


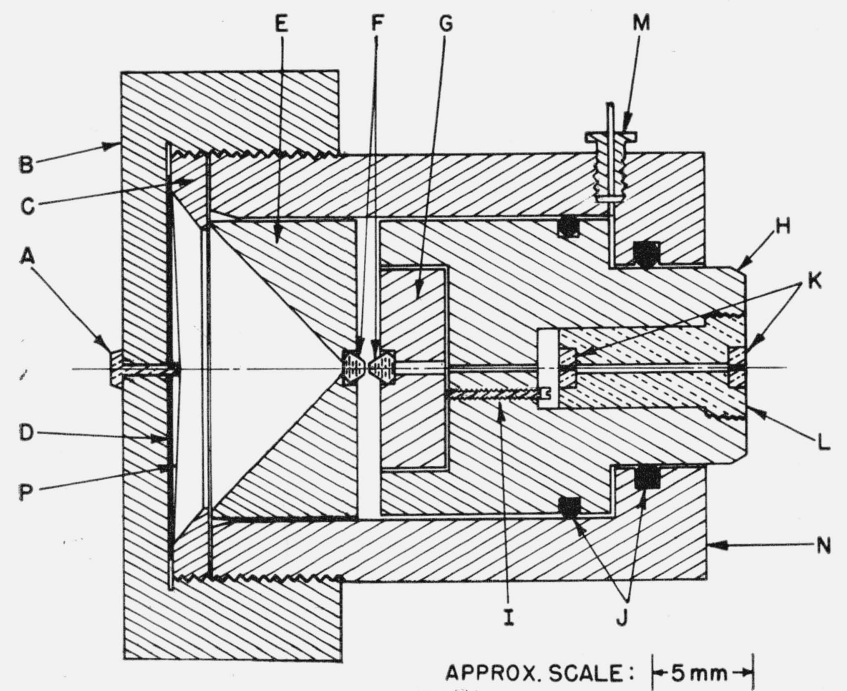

Figure 1. Cross section of high pressure X-ray powder camera.

diamonds are seated on their table faces in small close-fitting recesses. The diamond in the piston is seated in a small cylindrical insert $(G)$ which fits into the piston. The culets of both diamonds were ground off to produce small flat surfaces parallel to the tables. The flat-topped steel screw (I) in the piston is one of three such screws spaced $120^{\circ}$ apart about the axial hole of the piston to facilitate alinement of the flat surfaces of the two diamonds when necessary. The X-ray beam enters through the collimating pinholes, 0.014 in. in diameter, which are contained in the brass inserts $(\mathrm{K})$. The small axial hole in the piston $(\mathrm{H})$ acts as a guard slit which eliminates diffraction lines originating in the pinholes.

In use zirconium-filtered molybdenum radiation traverses both diamonds and the film of powder compressed between the diamond surfaces. Diffraction rings from the powdered specimen diverge through the diamond in the entablature and are recorded on the film (D), placed inside the cap and held securely between cap and thrust washer. In all experiments the film is covered with a lightprotective paper $(\mathrm{P})$. A small beam stop $(\mathrm{A})$ is inserted through the cap and secures the film at the center. The entablature is machined out to produce a conical surface of semivertex angle of approximately $45^{\circ}$.

From figure 1 it is apparent that the specimen is not located within the cone obtained by extending the inner conical surface of the entablature that is designed to serve as the exit aperture. The distance from the specimen to the apex of this cone for a given entablature, is determined by the thickness of the diamond. In these experiments the diamond is of such thickness that the specimen is always above the apex of the cone and the effective aperture is defined by the cone formed by the specimen and the aperture in contact with the diamond table surface. Under the experimental conditions used here, diffraction rings with $2 \theta>20^{\circ}$ are not transmitted.

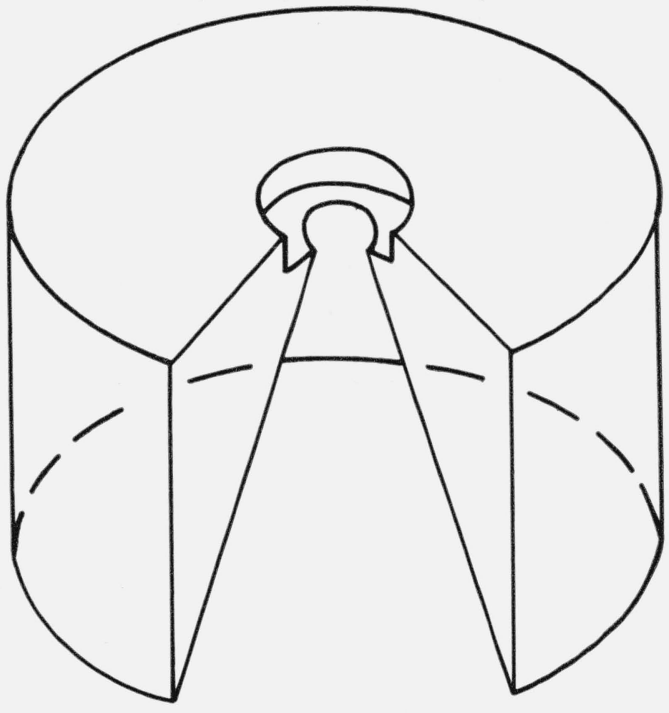

Figure 2. Details of entablature.

Two solutions to this problem were attempted. First the diamond was made thinner thus bringing the specimen plane within the cone formed by extending the conical surface of the entablature. For low pressure work this was found to be useful. High pressures could not be obtained in this manner because of the reduction in the strength of the diamond. Second, a wedge was cut out of the entablature (see fig. 2). This permitted higher-angle rings to be diffracted through the wedge up to $2 \theta \approx 35^{\circ}$. However, a portion of the diamond seat and retaining wall were removed by this process, and at high stresses some lateral motion of the diamond was observed. A less radical procedure consisted in removing only a small portion of the diamond seat and leaving the girdle-retaining wall intact. By using a hardened steel entablature in this manner, measurements have been made to pressures of at least 60 kilobars with no evidence of motion of the diamond. The photographs under these circumstances show the higher angle rings as arcs while those of lower angle appear as complete circles. The complete rings permit centering the film for measurement of the diameters of the ares.

In figure 1 it will be noted that the pressure must be released before the photographic film can be removed for processing. Another short-coming is associated with the inconveniently short specimento-film distance. Greater precision in the measurement of the diffraction-ring diameters can be achieved by increasing this distance. A bell-shaped film enclosure was made for optional use to permit locating the film at distances of from $30 \mathrm{~mm}$ to $75 \mathrm{~mm}$ from the specimen (see fig. 3). This closure (R) screws onto a modified cap $(\mathrm{S})$ which is bored out to permit exit of the diffraction rings. The modified cap contains a thrust washer (T). A cylindrical spacer (U) provides a shoulder on which the film plate $(\mathrm{V})$ containing the film $(\mathrm{X})$ and light-protective paper (Y) rests. The film and paper are held fixed 
by the taut beam stop (W) and film plate (V). This entire unit shown in figure 3 replaces the retaining cap (B) and thrust washer (C) shown in figure 1. To change the film the bell-shaped enclosure $(\mathrm{R})$ is unscrewed from the retaining cap $(\mathrm{S})$ and taken as a unit to the dark room. The film plate (V) is unscrewed from the bell-shaped housing. The film is removed from the film plate by unscrewing the beam stop (W). This arrangement permits repeated exposures at constant pressure, and also allows diffraction patterns to be obtained with different pressures without the necessity of releasing the pressure between runs.

Both arrangements have desirable characteristics. In this study the latter modification was usually preferred because the resulting larger X-ray powder pattern was easier to analyze. The error in the diffraction ring measurement was thereby reduced. Although the longer specimen-to-film distance increases accuracy, it also increases exposure time required 4 -fold. The increased exposure time is undesirable because prolonged periods of high pressure on the specimen have been suspected of causing small cracks to develop in the pressure surface of the larger faced diamond. The cracks may have developed because of poor alinement of the two diamond pressure surfaces. Experience [14] has shown that better alinement reduces the cracking tendency and increases the useful life of the diamonds considerably. One set of diamonds with properly alined pressure surfaces, for example, was used daily for about a month to compress materials to pressures up to 30,000 bars before one of the diamonds developed very shallow cracks approximately $0.05 \mathrm{~mm}$ long. Even with these flaws the set of diamonds contiriued to perform satisfactorily. On continued use, however, the cracks increased in size and in the study of bismuth some material was forced into these cracks. The bismuth apparently was not removed by the cleaning technique employed, and as a result, in the subsequent study of potassium iodide at 20 kilobars, there was interference from bismuth in the X-ray powder pattern. This problem is considered further in the discussion of potassium iodide.

In all experiments pressures were generated by means of a commercial hand pump using oil as the hydraulic liquid. The pressure was measured with a bourdon-tube gage. A valve was mounted in the liquid line between the pump and the gage so that the pressure cell and gage could be isolated from the pump during exposures. The dimensions of the unit and the diamond surface areas are such that the hydraulic pressure is magnified by a factor of approximately 500. Thus to achieve a pressure of 60 kilobars on the specimen a liquid pressure of approximately 120 bars is required.

\section{Method}

The materials studied were silver iodide, potassium iodide, bismuth, and thallium. They were selected because: (1) the pressures at which their known

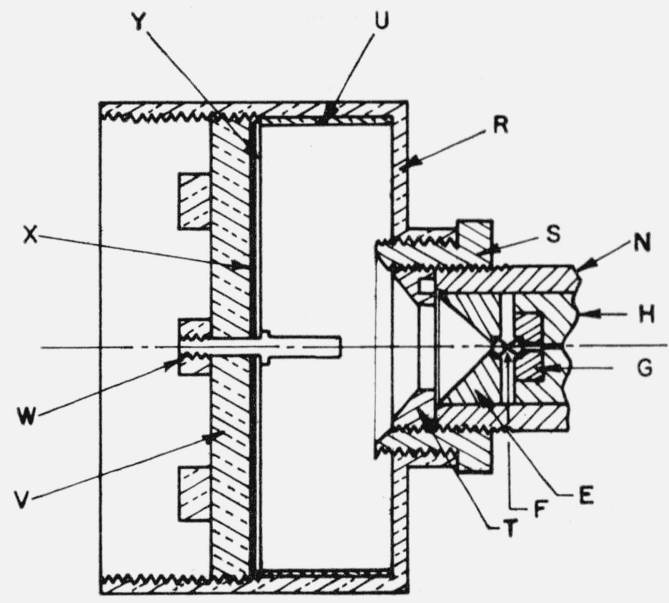

SCALE: $\rightarrow 20 \mathrm{~mm}$

Figure 3. Cross section of modified film enclosure.

transitions occur cover a wide range [1, 15], and (2) structural data on silver iodide and potassium iodide are available for comparison $[4,5,9]$. The materials were commercially available chemicals and were used in this study with no further purification. Silver iodide consisted initially of a mixture of the hexagonal and cubic forms and was converted predominantly to the metastable cubic form by grinding vigorously for about $1 / 2 \mathrm{hr}[16]$.

About $5 \mathrm{mg}$ of the powdered material was placed on the face of the diamond in the fixed piston. The material on the surface of the diamond was tamped lightly with a spatula. Some adhered to the diamond face; the excess was removed. The small patch of material remaining on the diamond face served as the sample. The thallium specimen, however, was not prepared in this manner. Instead, a thin film of freshly sliced metal was placed between the two diamond pressure faces and quickly compressed to minimize contact with the air.

The entablature containing the specimen was inserted in the piston housing of the mounted and alined camera. The retaining cap containing the film, the light-protective paper, and the thrust washer was screwed on tightly. A pressure of 500 bars was slowly applied to the specimen to create a uniform film and also to bring all moving components into their proper positions. The pressure was reduced over a $5 \mathrm{~min}$ period to a value just greater than atmospheric pressure. By keeping a slight positive pressure on the specimen, all the moving components in the camera were properly positioned. The X-ray powder patterns of the low pressure phases were obtained under these conditions.

The X-ray powder patterns of the high pressure form were obtained as follows. After the low pressure pattern was obtained, new film was placed in the camera and the pressure was raised to a value approximately 20 percent above the transition pressure determined by Bridgman $[1,15]$ for the specimen being studied. The pressure was then reduced 
slowly to a value slightly greater than the reported value for the transition. The reduction in pressure was carried out slowly over a period of $5 \mathrm{~min}$. The high pressure powder pattern was then obtained.

It must be emphasized that there was no effort made to determine accurate transition pressures for these materials. The object was to determine if a transition could be detected by this technique, and what structurally significant crystallographic data could be obtained. The pressures were calculated from the fluid pressure, the force on the sliding piston, and the area of the smaller of the two diamond pressure surfaces.

The nature and accuracy of the pressures calculated from the load applied and the area of the smaller diamond surface have been discussed previously in connection with the infrared cell [14]. In the spectroscopic studies it was noted that the observed transition of sodium nitrite near 15 kilobars agreed with previously reported data within the experimental error. Additional experiments were performed to determine the initial appearance of the bismuth transition in the present apparatus. These studies showed that the transition was first noted between calculated pressures of 19.5 and 21.0 kilobars, values which are considerably lower than the pressure of approximately 25 kilobars reported by Bridgman [1]. The discrepancy between the spectroscopic and X-ray results has not been investigated but is believed to arise predominantly from errors arising in this work. Significant errors in pressure may be caused by the following factors: (1) The magnitude of the pressure gradients and the effect of the topography of the diamond surfaces on the gradients; (2) the effect of alinement errors on the pressure gradients; and (3) the effect of the film thickness and shear strength of the specimen. It is believed also that the effective area of the smaller diamond used here is somewhat less than the measured area because of departures of its surface from optical flatness.

The specimen-to-film distance was determined by calibration and also by measurement. In the calibration technique, a powder pattern obtained under the conditions specified above for the low-pressure phase study, was used to calculate the specimen-tofilm distance using " $d$ "- spacings obtained by Swanson et al., [17]. The calculated value, which differed only slightly from the measured value, was used in the interpretation of the powder patterns of the high pressure phases. A check on the specimento-film distance was made to determine if any permanent distortion occurred after application of high pressure. This was done by calibrating the instrument before and after high pressures had been applied. No significant change was observed. The diameters of the diffraction rings were measured to $0.05 \mathrm{~mm}$ with a hair-line type instrument. The incomplete rings were measured by a difference technique with reference to a complete inner ring.

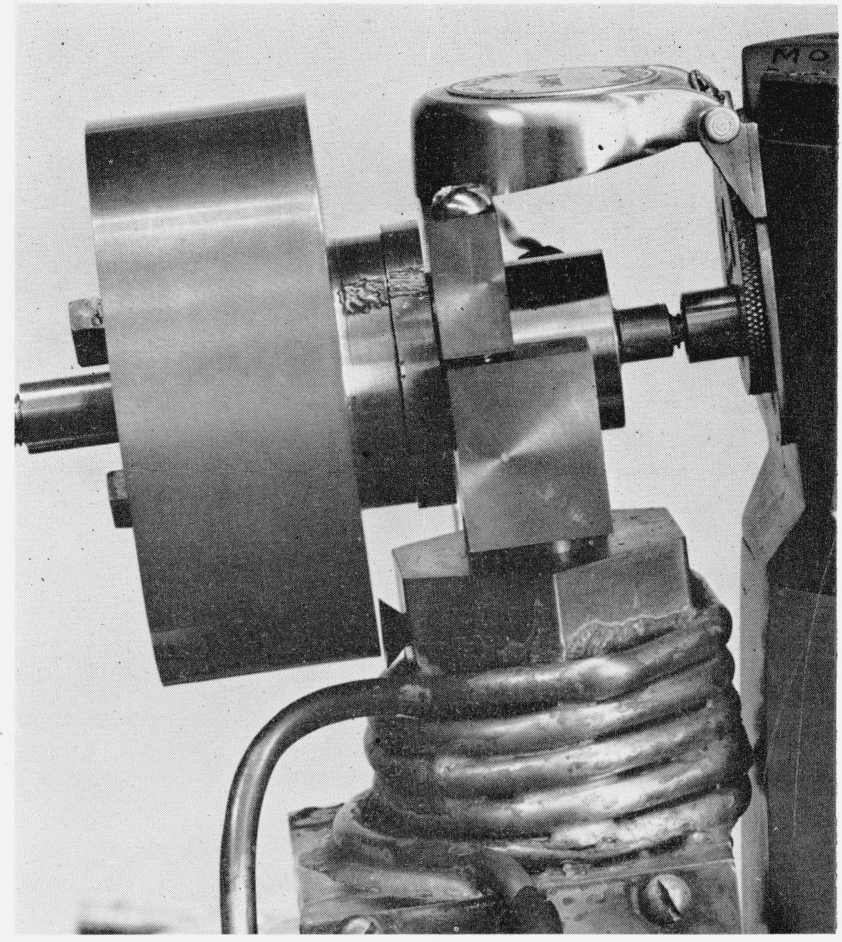

FiguRE 4. Photograph of modified high pressure X-ray powder camera in operation.

Silver iodide was studied using the original design of the instrument, which prevented film replacement unless the pressure on the specimen was released. For this arrangement a specimen-to-film distance of $16.13 \mathrm{~mm}$ was calculated. Using zirconium-filtered molybdenum radiation ( $45 \mathrm{kv}$ and $20 \mathrm{ma}$ ), a film exposure of $1 \mathrm{hr}$ was required for a satisfactory powder pattern. The remaining materials were studied with the modified instrument described earlier in this report. This camera had a $52.6 \mathrm{~mm}$ specimen-to-film distance and required an exposure of $4 \mathrm{hr}$ with the same radiation source. A photograph of the latter instrument in operation is shown in figure 4.

\section{Results}

\subsection{Silver Iodide}

Two halves of X-ray powder patterns of silver iodide obtained with this camera are shown in figure 5. The left half refers to material studied at approximately 1 bar; the right half to material studied at approximately 3,300 bars. The two films were split and matched for easy comparison. Except for pressure the experimental conditions in both exposures were the same. Diffraction rings are clearly visible and a transition is quite apparent. This is expected because Bridgman [15] showed that silver iodide exhibits a transition at about 3,000 


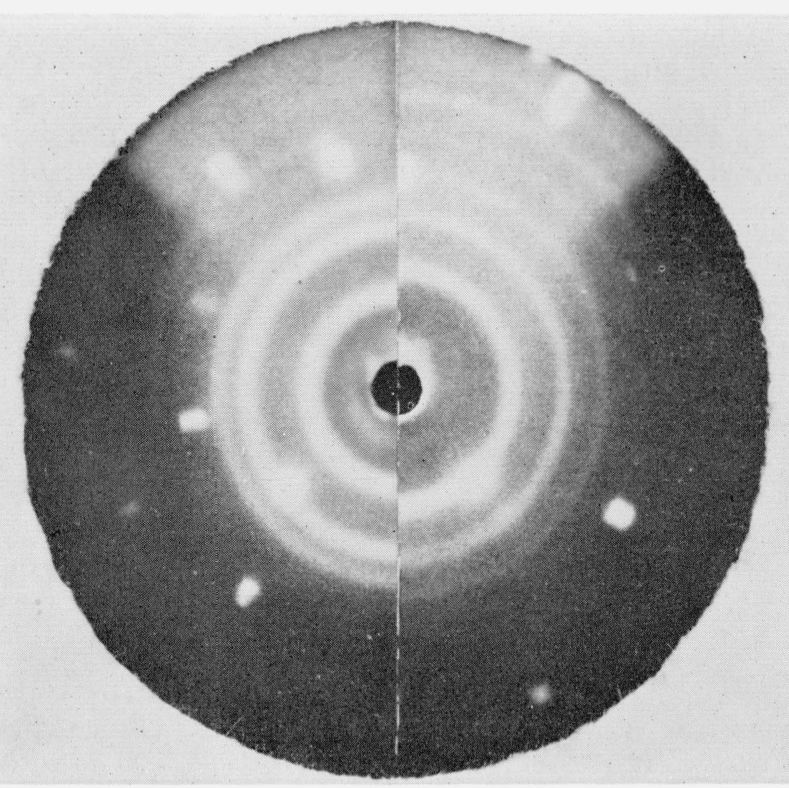

Figure 5. Matched halves of X-ray powder patterns of AgI (left half, AgI at apprsx. 1 bar; right half, AgI at approx. 3.8 kilobars).

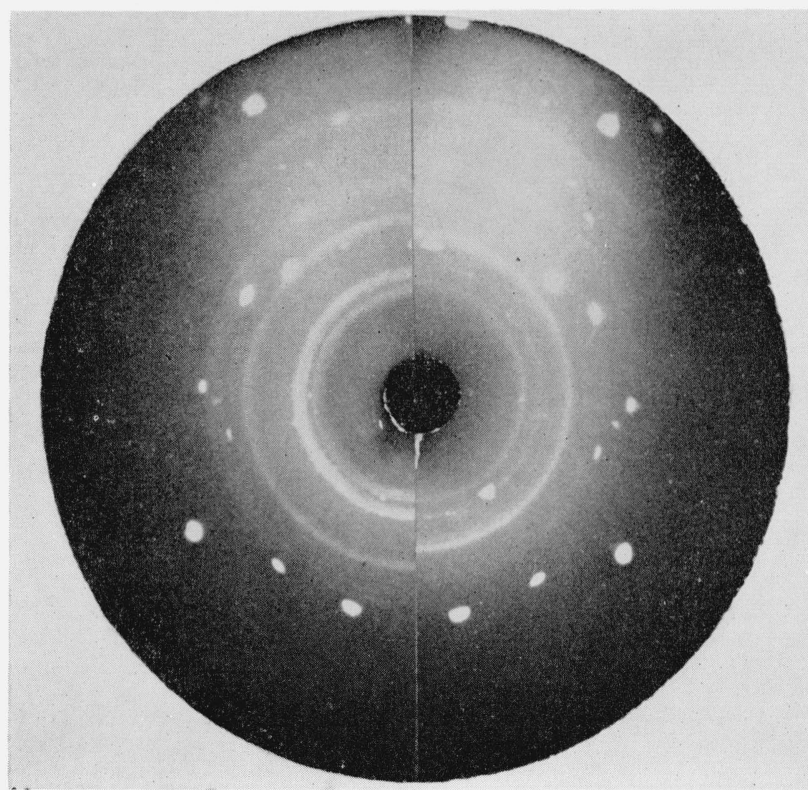

FIgure 6. Matched halves of X-ray powder patterns of KI (left half, KI at approx. 1 bar; right half, KI at approx. 20 kilobars).

bars. The incomplete rings are due to the wedge cut-out in the entablature. A close examination of the film indicates no evidence of low-pressure material contributing to the powder pattern on the right. It follows that the minimum pressure in the region penetrated by the radiation beam was less than approximately 300 bars below the calculated pressure. The importance of pressure gradients is recognized in determining the pressure on the speci-

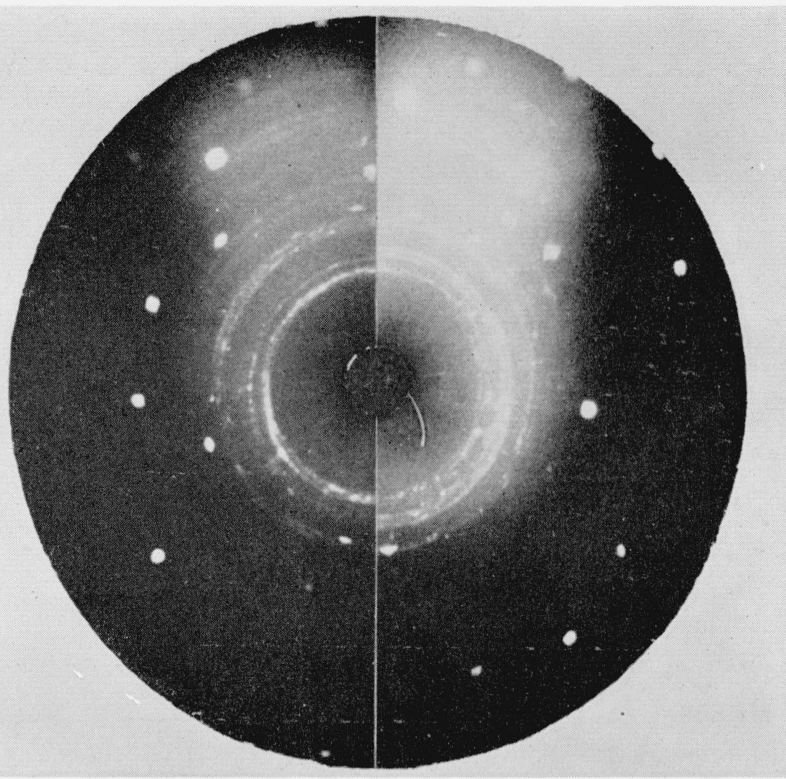

Figure 7. Matched halves of X-ray powder patterns of $\mathrm{Bi}$ (left half, Bi at approx. 1 bar; right half, Bi at approx. 28 kilobars).

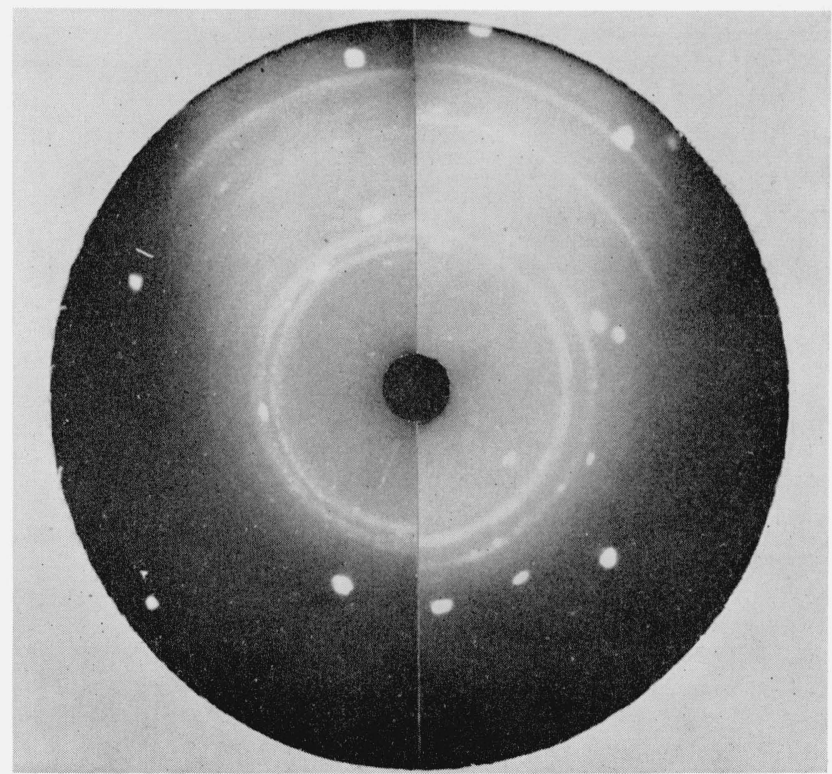

Figure 8. Matched halves of X-ray powder patterns of $\mathrm{Tl}$ (left half, Tl at approx. 1 bar; right half, Tl at approx. 60 kilobars).

men. The spots on the film are Laue spots originating from the two diamonds.

The six rings in the high pressure phase pattern were readily indexed as face-centered cubic (see table 1). The high pressure form has the halitetype structure containing 4 molecules per unit cell. Comparison of these results with those obtained by Jacobs [4] shows good agreement. His average lattice constant is $6.070 \mathrm{~A}$. The calculated density 
of silver iodide III based on the present $6.067 \mathrm{~A}$ lattice constant obtained at 3,300 bars is $6.98 \mathrm{~g}$ $\mathrm{cm}^{-3}$. The total volume change calculated between 1 bar and 3,300 bars including the transition $\mathrm{AgI}-\mathrm{II} \rightarrow \mathrm{AgI}-\mathrm{III}$ is 18.5 percent. Using Bridgman's data [15] the corresponding volume change is estimated to be 18 percent.

\section{TABLE 1.- "d"-Spacings and lattice constants of AgI at approximately 3.3 kilobars}

\begin{tabular}{c|c|c}
\hline \hline$h k l$ & "cd"-Spacings, A & $\begin{array}{c}\text { Lattice } \\
\text { constant, A }\end{array}$ \\
\cline { 3 - 3 } 200 & 3.031 & 6.062 \\
220 & 2.158 & 6.103 \\
222 & 1.764 & 6.110 \\
400 & 1.507 & 6.028 \\
420 & 1.352 & 6.046 \\
422 & 1.241 & 6.080 \\
& &
\end{tabular}

Statistical average of lattice constant__._ $6.067^{*}$ Standard deviation 0.015

*See appendix.

\subsection{Potassium Iodide}

According to Bridgman [15] potassium iodide which normally exists in the $\mathrm{NaCl}$ type structure exhibits a transition at about 17,850 bars. Figure 6 demonstrates the transition. The modified instrument using a larger specimen-to-film distance was employed. Therefore, the films are different in appearance from those shown in figure 5. There are weak lines in these patterns which cannot be brought out more clearly by longer exposure because of a disproportionate increase in background. This background is believed due to scattering from the two diamonds. Results from this study are shown in table 2 . The high pressure form was readily indexed as simple cubic, that is, the $\mathrm{CsCl}$ type structure. Satisfactory agreement with Jamieson's results [9] is obtained. He reported an average lattice constant of $4.13 \mathrm{~A}$ at 20,800 bars. Using the $4.093 \mathrm{~A}$ value obtained in these studies, the calculated density for potassium iodide II at approximately 20 kilobars is $4.02 \mathrm{~g} \mathrm{~cm}^{-3}$. A volume change of 22.2 percent is calculated for potassium iodide over the pressure range 1-20,000 bars including the transition KI $-\mathrm{I} \rightarrow \mathrm{KI}$ - II. A corresponding value of 20 percent is estimated from Bridgman's data [15].

\section{TABLE 2.- "d"-Spacings and lattice constants of $K I$ at approximately 20 kilobars}

\begin{tabular}{c|c|c}
\hline \hline$h k l$ & "d"-Spacing, A & $\begin{array}{c}\text { Lattice } \\
\text { constant, A }\end{array}$ \\
\cline { 2 - 3 } 100 & 4.037 & 4.037 \\
110 & 2.902 & 4.103 \\
111 & 2.361 & 4.089 \\
200 & 2.056 & 4.112 \\
210 & 1.829 & 4.090 \\
211 & 1.670 & 4.090
\end{tabular}

Statistical average of lattice constant__.. 4.093* Standard deviation.................... 0.008

*See appendix
A close examination of the high pressure powder pattern reveals a diffraction ring (second from center) which is not due to potassium iodide. It is believed that this ring is due to bismuth which had been forced into some small cracks in the diamond face. The ordinary means employed in cleaning the diamond faces did not remove the material. Later, new diamonds were prepared and studies on potassium iodide with these new diamonds showed no evidence of this ring.

\subsection{Bismuth}

Initial studies on bismuth revealed a complex X-ray powder pattern for the $m$ a terial at approximately 28 kilobars. This is demonstrated in figure 7. Due to its complexity the pattern could not be indexed. The " $d$ "'-spacings obtained from the film on the right are listed in table 3 . Upon examination, one strongly suspects that the room pressure hexagonal form is present. This is entirely possible. The fact that bismuth exhibits two very close transitions, one at about 24 kilobars, and the other at about 27 kilobars adds to the problem [18]. Both high pressure forms could conceivably be present simultaneously. Consequently, no conclusions were drawn about the high pressure form of bismuth at 28 kilobars. Further work to solve this problem is contemplated.

\section{TABLE 3. X-ray powder data for Bi at approximately} 28 kilobars

\begin{tabular}{c|c}
\hline \hline Angle, 20, degrees & " $d$ "'-Spacing, A \\
\cline { 2 - 2 } & \\
\hline 12.40 & 3.284 \\
13.72 & 2.969 \\
14.38 & 2.833 \\
15.83 & 2.561 \\
17.10 & 2.385 \\
18.40 & 2.218 \\
20.60 & 1.983 \\
22.45 & 1.823 \\
\end{tabular}

\subsection{Thallium}

Thallium exhibits a transition reported at about 40 kilobars [19]. Figure 8 demonstrates the transition, showing the high pressure form at approximately 60 kilobars. This phase has apparently never before been observed by X-ray diffraction. Table 4 gives the analysis of the high pressure pattern. It was readily indexed as face-centered cubic. Based on the lattice constant $4.778 \mathrm{~A}$, a density of $12.44 \mathrm{~g} \mathrm{~cm}^{-3}$ for thallium at about 60 kilobars was calculated. The total volume change calculated including the transition $\mathrm{Tl}_{\text {h.c.p. }} \rightarrow \mathrm{Tl}_{\text {f.c.c., }}$ is 5.0 percent. The corresponding volume change based on Bridgman's data [19] is estimated to be 10 percent.

The results of this study corroborate two predictions which have been made for the high pressure form of thallium. Based on the correlation of thermodynamic data at one atmosphere with high pressure equilibria, Kaufman [20] has predicted a facecentered cubic structure for thallium. A similar prediction has been made by Parthe [21] using a space-filling concept. 
TABLE 4. "d"-Spacings and lattice constants of $\mathrm{Tl}$ at approximately 60 kilobars

\begin{tabular}{c|c|c}
\hline$h k l$ & "d"-Spacing, A & $\begin{array}{c}\text { Lattice con- } \\
\text { stant, A }\end{array}$ \\
\cline { 3 - 3 } & & \\
\hline 111 & 2.731 & 4.730 \\
200 & 2.391 & 4.782 \\
220 & 1.689 & 4.776 \\
311 & 1.442 & 4.783 \\
222 & 1.383 & 4.791 \\
\hline
\end{tabular}

Statistical average of lattice constant 4.778 * Standard deviation

*See appendix.

\section{Appendix}

It is desirable to weight the data statistically to obtain a more significant value of the lattice parameter. This is particularly important in these experiments because the diffraction rings are limited to low angles. The following method was used to determine the average lattice parameter and its standard deviation:

If $r$ represents the radius of a given ring, $D$ the specimen to film distance, and $2 \theta$ the diffraction angle, then when

$$
r<D \text { (as in these experiments) } \sin \theta \approx \frac{r}{2 D} .
$$

If the experimental error in measurement is $\epsilon$, then $\epsilon_{\sin \theta}=\frac{\epsilon_{r}}{2 D}$, where the subscripts denote the quantities to which the error refers. The error in the radius measurement is not a function of $r$.

The Bragg relationship for the spacing including the error term is, $\sin \theta=\frac{\lambda}{2 d}+\frac{\epsilon_{r}}{2 D}$, where $d$ is the interplanar spacing and $\lambda$ the wavelength.

For the cubic system, this may be written in terms of the lattice parameter, $a_{0}$, and the Miller indices, $h, k, l$, as

$$
2 D \sin \theta=\frac{D \lambda \sqrt{h^{2}+k^{2}+l^{2}}}{a_{0}}+\epsilon_{r} .
$$

(Paper 66A4-167)
Equation (2) contains a relationship between the two variables $2 D \sin \theta$ and $D \lambda \sqrt{h^{2}+k^{2}+l^{2}}$ representing a line through the origin of slope $1 / a_{0}$. The best value of $a_{0}$ was determined by least squares and the standard deviation by well-known methods (for example, see p. 42 of "Statistical Methods for Chemists" by W. J. Youden, John Wiley \& Sons, New York, N.Y., 1951).

The authors are indebted to the Institute of Geophysics of the University of California at Los Angeles for the use of their facilities in the preliminary developments of the X-ray camera. In particular, the assistance and advice of L. H. Adams and W. Hoffman are greatly appreciated. The authors are also indebted to J. Mandel of the National Bureau of Standards staff for advice on the statistical evaluation of the lattice parameters.

\section{References}

[1] P. W. Bridgman, The physies of high pressure (G. Bell \& Sons Ltd., London, 1952) ch. VIII.

[2] R. L. MeFarlan, J. Chem. Phys. 4, 60 (1936).

[3] R. L. McFarlan, J. Chem. Phys. 4, 253 (1936).

[4] R. B. Jacobs, Phys. Rev. 54, 325 (1938).

[5] R. B. Jacobs, Phys. Rev. 54, 468 (1938).

[6] A. W. Lawson and N. A. Riley, Rev. Sci. Instr. 20, 763 (1949).

[7] A. W. Lawson and T. Y. Tang, Rev. Sci. Instr. 21, 815 (1950).

[8] J. C. Jamieson, Z. Krist. 10\%, 65 (1956).

[9] J. C. Jamieson, J. Geol. 65, 334 (1957).

[10] J. C. Jamieson, A. W. Lawson, and N. D. Nachtrieb, Rev. Sci. Instr. 30, 1016 (1959).

[11] J. C. Jamieson, in "Progress in very high pressure research" (Proceedings of an international conference held at Bolton Landing, Lake George, New York, June 13-14, 1960, John Wiley \& Sons, Inc., New York, N.Y., 1961).

[12] L. H. Adams (private communication).

[13] V. V. Evdokimova and L. F. Vereshchagin, Soviet Physics Solid State. English Translation 2, 1539 (1961).

[14] C. E. Weir, E. R. Lippincott, A. Van Valkenburg, and E. N. Bunting, J. Research NBS 63A, 55 (1959); Spectrochim. Acta 16, 58 (1960).

[15] P. W. Bridgman, Proc. Amer. Acad. of Arts and Sci. 76, 1 (1945).

[16] N. H. Kolkmeijer and J. W. A. van Hengel, Z. Krist. 88, 317 (1934).

[17] H. E. Swanson et al., Standard X-ray diffraction powder patterns, NBS Cir. 539, Vol. 1-10.

[18] F. P. Bundy, Phys. Rev. 110, 314 (1958).

[19] P. W. Bridgman, Phys. Rev. 60, 351 (1941).

[20] L. Kaufman, Acta Met. 9, 896 (1961).

[21] E. Parthé, Z. Krist. 115, 1/2. 52 (1961). 